\title{
Which Neisseria?
}

\author{
R. R. WILLCOX, R. C. SPENCER, AND C. ISON \\ From St Mary's Hospital, London and King Edward VII Hospital, Windsor
}

SUMMARY A case of disseminated gonococcal infection in which the gonococcus was isolated from the blood and genitalia and the meningococcus from the throat is described. A second patient, in whom the meningococcus was isolated from the throat and the gonococcus from the genitals but in whom no organisms were recovered from the blood, had lesions resembling those of a disseminated infection. The results of 150 throat cultures from patients who were examined at a clinic because they had a history of oral sexual intercourse are presented. The meningococcus was isolated nearly six times more often in patients with genital gonorrhoea than in those in whom genital cultures were negative, while the gonococcus was found $2 \frac{1}{2}$ times more often in those who carried the meningococcus in the throat than in those who did not. If these findings can be confirmed it could indicate an individual susceptibility to the acquisition of Neisserian organisms that would merit further investigation.

\section{Introduction}

At one time it was thought that confusion between the two pathogenic Neisseria seldom occurred as each seemed to keep to its own territory, the meningococcus to the nasopharynx and the gonococcus to the genitals. However, with improvements in cultural technique and the increased practice, or recognition, of orogenital intercourse the gonococcus today is recovered from the mouth while the meningococcus may occasionally be isolated from the genitourinary tract in men and women (Murray, 1939; Carpenter and Charles, 1942; Feldman, 1971).

Both organisms can evoke a strikingly similar picture should either be involved in a septicaemic state, and a definite diagnosis can be made only by the bacteriologist. Indeed a septicaemia has been reported with Neisseria meningitidis when the organism was found in the endocervix (Keys et al., 1971). Moreover the gonococcus can occasionally cause meningitis (Ikejiani, 1958; Taubin and Landsberg, 1971; Handsfield, 1975) and it may well be overlooked unless sugar fermentation tests are done. This paper reports two cases of disseminated Neisserian infection in which both pathogens were recovered from the local sites.

Paper presented at the meeting of the Medical Society for the Study of Venereal Diseases, Vienna, 5-8 May 1977

Address for reprints: R. R. Willcox, MD, FRCP, Consultant Venereologist, St Mary's Hospital, London W2

Received for publication 17 June 1977
CASE 1

The first patient was a 23-year-old Moroccano chambermaid. There was a history of treatment for possible tuberculosis when she had been five-years old and her mother had died from tuberculosis.

In October 1976, after the insertion of an intrauterine contraceptive device a month previously which had been changed to a copper coil one week before, she was admitted to the gynaecological department of a London hospital with pelvic inflammatory disease. She was found to be tender in both iliac fossae with rebound tenderness, and both Fallopian tubes were enlarged and tender. The coil was removed and she was initially given metronidazole (Flagyl) and clotrimazole (Canesten), pending the report on investigation of a high vaginal swab. This showed gonococci but no trichomonads. She was then treated with phenoxymethyl penicillin plus sulphadimidine by mouth for four and a half days.

She was re-admitted through the Accident and Emergency Department late on 19 March 1977. She complained of productive cough for one week with a little haemontysis the previous day. There had been a poor appetite and some lower abdominal pain for seven days during which time a number of joints-namely, both shoulders and wrists, left ankle and the metacarpophalangeal joints of both handshad become painful.

Since the previous day she had complained of a headache and a rash which began on the hands, 
then spread to the arms and feet, followed by the legs. It was just beginning to show on the abdomen.

The patient was pale and she spoke little English. The rash consisted of painful, non-irritant, pink macules with dark centres, the older lesions being much larger with a central necrotic area and surrounding erythema (Figure). The lesions were particularly developed on the hands and feet and were just starting on the abdomen: the face and back were clear. There was a slight swelling of the metacarpophalangeal joints of both hands.

The temperature was $36.7^{\circ} \mathrm{C}$, the pulse $100 / \mathrm{min}$, and nothing abnormal was found in other systems.

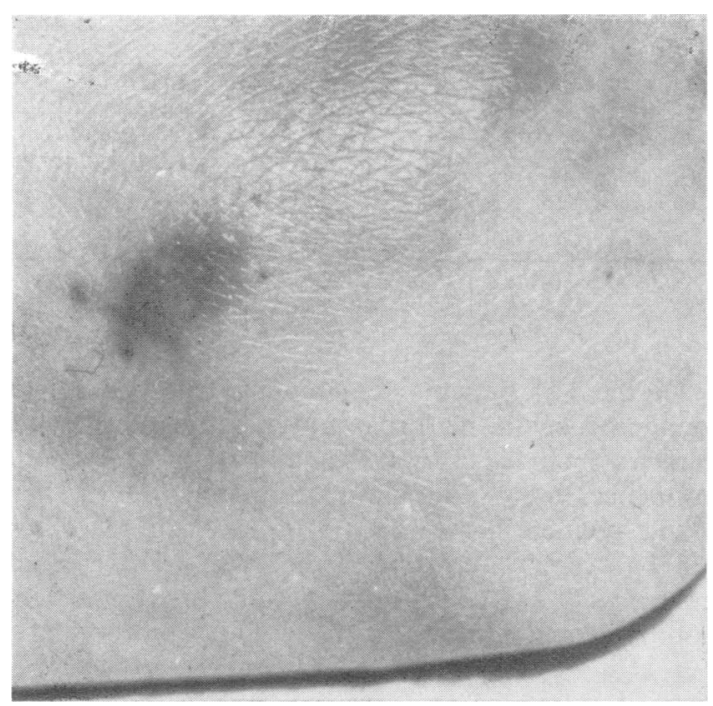

Figure Appearance of skin lesions.

\section{Investigations}

The chest $x$ ray revealed a 'slight increased prominence in chest markings'. Haemoglobin was $10.9 \mathrm{~g} / \mathrm{dl}$ $(10.9 \mathrm{~g} / 100 \mathrm{ml})$, red cell count $3.6 \times 10^{12} / 1(3.6 \times$ $\left.10^{6} / \mathrm{mm}^{3}\right)$. The white cell count was $13 \times 10^{9} / 1(13 \times$ $10^{3} / \mathrm{mm}^{3}$ ) with a normal differential (polymorphs $72 \%$, lymphocytes $20 \%$, monocytes $6 \%$, and eosinophils $2 \%$ ).

The serological tests for syphilis read: Venereal Diseases Research Laboratory (VDRL) results positive neat (later negative), TPHA negative, FTA negative. A blood culture was reported as growing a coagulase-negative staphylococcus, probably a contaminant.

A preliminary diagnosis of vasculitis after viral infection was made and the opinion of a dermatologist was sought.

\section{Dermatologist's diagnosis}

The dermatologist first diagnosed gonococcal septicaemia and immediate referral to a venereologist was recommended. If the venereologist's findings should prove negative the following choices were suggested: meningococcal septicaemia, drug reaction, collagen disease.

\section{Venereologist's action}

At the Praed St Clinic the patient was questioned about sexual contacts but these were denied since the previous episode. Nothing very helpful was found on visual, manual, or bimanual examination apart from some vague lower abdominal discomfort. Smears were taken from the urethra and cervix and cultures were plated directly with material from the urethra, cervix, rectum, and mouth. A blood culture using gonococcal medium was also made.

Neisseria were at once found in the Gram-stained genital smear and the patient was therefore prescribed penicillin in the form of 1 megaunit four times daily intramuscularly plus co-trimoxazole (Septrin) two tablets twice daily for 12 days, and she was returned to the referring hospital. The initiation of treatment was followed almost immediately by some vomiting, headache, and a spike of fever to $39^{\circ} \mathrm{C}$ for which she was given chlorpheniramine (Piriton).

\section{Bacteriologist's techniques}

Blood was inoculated into a blood culture set comprising $50 \mathrm{ml}$ dextrose broth, $15 \mathrm{ml}$ of Robertson's cooked meat medium, and a modified Castaneda slope (Southern Group Laboratories, Lewisham). After 24 hours of incubation, subcultures were made on to Difco gonococcal medium base plus $20 \mathrm{ml}$ per litre of supplement $A$ and $2 \mathrm{ml}$ per litre of supplement B. Supplement A consisted of $20 \%$ glucose, $0.25 \% \mathrm{~L}$ glutamine, and $0.002 \%$ cocarboxylase; supplement $\mathrm{B}$ was $0.5 \%$ ferric nitrate.

Selective media are not used for cultures of blood, cerebrospinal, or synovial fluid as contaminating organisms are not a problem and a selective medium may hinder the growth of some gonococcal strains. On the other hand, throat and urethral specimens were plated directly on to selective mediumconsisting of, the culture medium already described plus vancomycin, colistin methane sulphonate, trimethoprim, and nystatin (Phillips et al., 1972).

All the plates were incubated at $37^{\circ} \mathrm{C}$ for up to 48 hours in a Lab-line carbon-dioxide incubator (Burkard Scientific Sales, Rickmansworth). Colonies of Neisseria gonorrhoeae and N. meningitidis were identified by the oxidase reaction, Gram stain, 
immunofluorescence, and sugar fermentation tests using four different Difco serum-free media containing glucose, sucrose, lactose, and maltose (Flynn and Waitkins, 1972).

\section{Results of cultures}

The patient was next seen two days later by which time the skin lesions were better. The culture from the genitals showed $N$. gonorrhoeae confirmed by immunofluorescence and sugars, and using similar methods, $N$. meningitidis was isolated from the throat. There has been no growth to date from the blood. The treatment was continued as before and by the time the patient returned four days later the blood culture showed $N$. gonorrhoeae confirmed by immunofluorescence and sugars.

\section{CASE 2}

In 1975 one of the authors (RRW) had a similar case in a young woman with genital gonorrhoea who was admitted to King Edward VII Hospital, Windsor with skin lesions resembling a disseminated infection, for which she was treated with large doses of penicillin. This patient also had meningococci in the throat but the blood culture was sterile.

It is thus evident that the presence of two different Neisserian pathogens in the same patient does not prevent the occurrence of a disseminated infection.

Frequency of simultaneous carriage of both organisms The results are reviewed of 150 throat cultures in patients who were examined because they had a history of oral sexual intercourse, or were requesting examination, or attending as contacts of patients with gonorrhoea or non-gonococcal urethritis during the three-week period from 14 March to 4 April 1977. One hundred and eighteen were men. There were 21 cases in which meningococci were found $(14.0 \%)$. Genital smears and cultures were also made. Of 76 cases in which cultures were negative meningococci were recovered from the throat in $5.3 \%$. In 26 men the urethral smears were negative but no cultures were performed. The incidence of meningococci in the throat of this group was marginally higher at $7 \cdot 7 \%$. However in the 48 cases in which both smears and cultures of genital material were taken in which one or both proved positive the incidence of meningococci in the throat was as high as $31 \cdot 3 \%$ (Table 1 ).

If the 26 cases in which the examination was incomplete are ignored, the findings were equally striking when considered in reverse (Table 2).
Table 1 Meningococci in throat related to gonococci in genitals

\begin{tabular}{lclc}
\hline $\begin{array}{l}\text { Genital smears } \\
\text { and cultures } \\
\text { for gonococci }\end{array}$ & No. tested & No. & $\%$ \\
\cline { 2 - 4 } $\begin{array}{l}\text { Both negative } \\
\begin{array}{l}\text { Incomplete } \\
\text { investigation }\end{array}\end{array}$ & 76 & 4 & $5 \cdot 3$ \\
$\begin{array}{l}\text { One or both } \\
\text { positive }\end{array}$ & 26 & 2 & $7 \cdot 7$ \\
\begin{tabular}{l} 
Total \\
\hline
\end{tabular} & 150 & 15 & $31 \cdot 3$ \\
\hline
\end{tabular}

Combining 'Both negative' and 'Incomplete investigation' $\chi_{1}{ }^{2}=17 \cdot 43$, $\mathbf{P}<0.001$

Table 2 Gonococci in genitals related to meningococci in throat

\begin{tabular}{llll}
\hline Throat & \multicolumn{2}{l}{ Genitals } & \\
\cline { 2 - 4 } & & \multicolumn{2}{c}{ Gonococcus positive } \\
\cline { 2 - 4 } & No. tested & No. & $\%$ \\
\hline Meningo positive & 19 & 15 & 79.0 \\
Meningo negative & 105 & 33 & 31.4 \\
Total & 124 & 48 & 38.7 \\
\hline
\end{tabular}

$\chi_{2}{ }^{1}=15 \cdot 30 P<0.001$

Of the 124 patients remaining, $19(15 \cdot 3 \%)$ ha $\$$ meningococci in the throat and these meningococcal carriers included 15 of the 48 patients in whon gonococci were found in the genitals $(31.3 \%)$. While gonococci were recovered in 48 of 124 cases $(38.7 \%)$ they were found in no fewer than 15 of 19 cases $(79 \%)$ of meningococcal throat carriers compared with $31.4 \%$ of those who were not.

\section{Discussion}

The $14 \%$ pharyngeal meningococcal carriage rate equates with $17 \cdot 2 \%$ found by Wiesner et al. (1973) in Seattle. It is fairly common for both meningococci and gonococci to be carried by the same patient. Indeed from these data there would appear to be some evidence that those who carry one Neisseria have a greater susceptibility to others of the same species.

\section{References}

Carpenter, C. M., and Charles, R. (1942). Isolation of the meningococcus from the genito-urinary tract of seven patients. American Journal of Public Health and the Nation's Health, 32, 640-643.

Feldman, H. A. (1971). Meningococcus and gonococcus: Never the twain . . . well hardly ever. New England Journal of Medicine, 285, 518-520.

Flynn, J., and Waitkins, S. A. (1972). A serum-free medium for testing fermentation reactions in Neisseria gonorrhoeae. Journal of Clinical Pathology, 25, 525-527. 
Handsfield, H. H. (1975). Disseminated gonococcal infections. Clinical Obstetrics and Gynecology, 18, 131-142.

Ikejiani, O. (1958). Gonococcal meningitis: A review of the literature and report of a case. West African Medical Journal, 7, 139-141.

Keys, T. F., Hecht, R. H., and Chow, A. W. (1971). Endocervical Neisseria meningitidis with meningococcemia. New England Journal of Medicine, 285, 505-506.

Murray, E. O. D. (1939). Meningococcal infections of the male urogenital tract and liability to confusion with gonococcus infections. Urologic and Cutaneous Review, 43, 739-741.
Phillips, I., Humphrey, D., Middleton, A., and Nicol, C. S. (1972). Diagnosis of gonorrhoea by culture on a selective medium containing vancomycin, colistin, nystatin, and trimethoprim (VCNT): A comparison with Gram staining and immunoflourescence. British Journal of Venereal Diseases, 48, 287-292.

Taubin, H. L., and Landsberg, L. (1971). Gonococcal meningitis. New England Journal of Medicine, 285, 504-505.

Wiesner, P. J., Tronca, E., Bonin, P., Pedersen, A. H. B., and Holmes, K. K. (1973). Clinical spectrum of pharyngeal gonococcal infections. New England Journal of Medicine, 288, 181-186. 